\title{
Comparison Of Intratracheal Administration Of Surfactant With Or Without Budesonide To Prevent Bronchopulmonary Dysplasia - A Parallel Randomized Pilot Study
}

\section{Kiran Vemireddy}

Rainbow Children's Hospital

Nalinikanta Panigrahy ( $\nabla$ nalini199@gmail.com )

Rainbow Children's Hospital https://orcid.org/0000-0003-4316-0517

Vijayanand Jamalpuri

Rainbow Children's Hospital

Alla Venkatlakshmi

Rainbow Children's Hospital

Dinesh Kumar Chirla

Rainbow Children's Hospital

\section{Research Article}

Keywords: bronchopulmonary dysplasia, budesonide, extreme preterm, pulmonary surfactant.

Posted Date: February 14th, 2022

DOI: https://doi.org/10.21203/rs.3.rs-1339070/v1

License: (c) (i) This work is licensed under a Creative Commons Attribution 4.0 International License.

Read Full License 


\section{Abstract}

Purpose : Despite improvements in preterm neonatal care, the incidence of bronchopulmonary dysplasia (BPD) has not decreased. Systemic glucocorticoids minimize BPD, but they may interfere with brain development. The impact of intratracheal budesonide along with surfactant on the incidence of BPD in extremely preterm infants is unknown.

Methods: This two arm parallel pilot trial over a period of 18 months recruited extreme preterm $(<28$ weeks) and extreme low birth weight (ELBW) neonates who were diagnosed with severe RDS (respiratory distress syndrome). Neonates were randomly allocated to one of two groups ( 54 intervention and 55 control). Intratracheal surfactant and budesonide were administered to the intervention group, while surfactant alone was administered to the control group.

Results: The study population had a mean gestational age of $26.1 \pm 0.2$ weeks and birth weight of 770.5 \pm 31.5 grams. Death ( RR 0.65 [0.30-1.38]; $p=0.267$ ) and combined BPD or death (RR 0.88 [0.73-1.06]; $p=$ 0.211 ) exhibited a non-significant decreasing trend; however, a significant reduction in the combined outcome of severe BPD or death (RR 0.57(0.33-0.97); $\mathrm{p}=0.040)$ was observed in the intervention group.

Conclusion: Intratracheal budesonide with surfactant administration is feasible. This may minimize severe BPD or death in extremely preterm infants with severe RDS without any harm.

\section{What Is Known}

- Bronchopulmonary dysplasia(BPD) is a major morbidity associated with extreme low birth weight (ELBW) and extreme preterm (<28 weeks' gestation) neonates.

- Post natal systemic steroids like dexamethasone and hydrocortisone can decrease incidence of BPD in this population but can harm by increasing poor neurodevelopmental outcome.

\section{What is New:}

- Combined intratracheal instillation of budesonide and surfactant in extreme preterm and ELBW neonates is feasible and safe.

- This early combination treatment may decrease severe BPD and death in extreme preterm neonates with RDS.

CTRI number - CTRI/2020/02/023310

\section{Introduction}

Bronchopulmonary dysplasia (BPD) is a multifactorial respiratory disorder caused by mechanical ventilation in premature neonates. BPD affects $10-40 \%$ of infants born before 28 weeks of gestation and weighing < $1000 \mathrm{gms}$ at birth (ELBW). [1] Early use of CPAP, vitamin A, and caffeine have been shown to 
reduce BPD.[2] No definitive medication can completely prevent BPD in neonates who require intubation or ventilation.

Lung inflammation and host immune responses are thought to be involved in BPD etiology. [3] Systemic glucocorticoids are anti-inflammatory and have been used to treat and prevent BPD but have negative neurological outcomes. [4] Using budesonide locally has advantages such as faster action, longer antiinflammatory effect, and lesser risk of systemic side effects. $[5,6]$ Inhaled budesonide can be used to treat BPD in preterm infants instead of systemic steroids. Early inhaled budesonide therapy reduces the incidence of BPD in preterm newborns owing to its significant topical effects. [7] Prior to the inflammatory and oxidative damage cascade, budesonide administration along with surfactant may help in the prevention of BPD.

Study by Yeh et al on very low birth weight (VLBW $<1500 \mathrm{gms})$ infants provides early evidence on the feasibility, safety, and potential benefit of giving corticosteroids directly into the lung via the intra-tracheal route during the acute phase of neonatal RDS. [8] The objective of this study was to see how intratracheal administration of surfactant combined with budesonide changed the incidence of BPD in a high-risk cohort of extreme low birth weight (ELBW $<1000 \mathrm{gms})$ and extreme preterm $(<28$ weeks) infants.

\section{Study Methods}

\section{Study population}

This unblinded randomized pilot study was conducted at Rainbow Children's Hospital's level 3 neonatal intensive care unit (NICU) in Hyderabad, India, from March 2020 to August, 2021. The inclusion criteria were decided within 6 hours after birth and included (1) inborn neonates with gestational age of (24 $27^{+6}$ ) weeks and birth weight $<1000 \mathrm{gms}(2)$ receiving mechanical ventilation through an endotracheal tube and a clinical decision to treat the infant with exogenous surfactant for RDS in less than 6 hours (3) obtaining prospective, written, informed parental/guardian consent (4) absence of major congenital anomalies or lethal cardiopulmonary disorder. These babies had a high risk of developing BPD. Verbal consent was obtained from parents before delivery anticipating delivery room surfactant and written consent within $6 \mathrm{~h}$ of birth.

\section{Surfactant and Budesonide regimens, dosages, and randomized procedures}

Block randomization with computer-generated permuted blocks of six was used, with half of the infants assigned to the intervention group and half to the control group. The intervention group received budesonide, in addition to the surfactant. The surfactant used in our investigation was curosurf 200 $\mathrm{mg} / \mathrm{kg}(2.5 \mathrm{ml} / \mathrm{kg}$ of an $80 \mathrm{mg} / \mathrm{ml}$ solution), manufactured by Chiesi Farmaceutici in Parma, Italy. As an intratracheal steroid (Intervention group), we utilised $0.25 \mathrm{mg} / \mathrm{kg}$ Budesonide $(0.5 \mathrm{mg} / 2 \mathrm{~mL}$ solution) Cipla, India along with surfactant. In an in vitro investigation employing a surfactometer and highperformance liquid chromatography, this mixture did not affect the biophysical and chemical properties of the surfactant. [9] The surfactant and budesonide mixture looks similar to a surfactant and does not 
discolour or crystallize. The study found no concerns regarding mixability. The syringe was gently vortexed before intratracheal instillation, and the surfactant or surfactant with budesonide mixture was delivered as usual. Repeat doses of surfactant with budesonide for infants in the intervention group and surfactant only for infants in the control group were given every 6-12 hours as per unit protocol.

\section{Neonatal Intensive care}

After delivery, a trial of nasal continuous positive airway pressure (NCPAP) was initiated for infants who exhibited respiratory distress immediately after birth. Infants with significant retraction, weak respiratory efforts, or apnea were intubated and randomized to surfactant only or surfactant with budesonide group. During the NICU stay, gentle ventilation was continued, and the neonate was extubated to continuous positive airway pressure (CPAP) as soon as possible. Blood gases were collected based on the clinical condition. Total fluid intake was initiated as ICU protocol. Infants with hemodynamically significant patent ductus arteriosus (HsPDA) were administered paracetamol. Postnatal systemic dexamethasone was reserved solely for newborns on positive pressure ventilation with a fio $2>40 \%$, even after 14 days of life. A course of dexamethasone with a low dosage protocol (DART regimen) was initiated once parental consent was obtained.[10]

\section{Outcome Measurements}

The primary outcome was death, BPD and combined death plus BPD. The Eunice Kennedy Shriver National Institute of Child Health and Human Development (NIH, Bethesda, MD) definition of BPD , which is severity based was used in our study.[11]. Mild BPD was defined as requiring supplemental oxygen therapy at 28 postnatal days but not at 36 weeks postmenstrual age. Moderate BPD requires less than $30 \%$ oxygen, while severe BPD requires at least $30 \%$ oxygen at 36 weeks postmenstrual.

Acute side effects, such as glucose and blood pressure changes, were assessed. Secondary outcomes included intraventricular hemorrhage (IVH), necrotizing enterocolitis (NEC), severe retinopathy of prematurity (ROP >stage 2), and clinical sepsis or bacteraemia. All included newborns had cranial ultrasound and ROP screening as part of the standard NICU protocol. Mortality, BPD morbidity, days of respiratory support, and repeat dose of surfactant were all studied.

\section{Statistical Analysis}

According to our past experience, approximately $50 \%$ of infants who met the inclusion criteria developed BPD or died. Power analysis was performed, assuming a reduction of BPD or death from $50 \%$ in the control group to $40 \%$ in the intervention group. A total of 770 patients were considered adequate for the study, with a $5 \%$ probability of type I error and a $20 \%$ chance of type II error.[12] Because this was a single centre pilot study, we could recruit 109 neonates in this study period of 18 months.

The data were collected using a standardized study proforma and transferred to MS-Excel for further analysis. The continuous variables were expressed as mean and standard deviation (SD) or median and Inter Quartile Range (IQR). The categorical variables were expressed as \% of frequency distribution. The 
two groups were compared by using either student's t test, median test, or Fishers exact test depending on the type of variables. All $p$ values are two sided, an association was considered significant if $p<0.05$. We reported relative risk (RR) with 95\% confidence interval. Statistical Package for Social Sciences (SPSS, version 27th ) was used for all statistical analysis. [13]

\section{Results}

Patient Population: A total of 191 neonates were screened for eligibility during the study period. In the first $6 \mathrm{~h}, 132$ neonates had severe RDS and required ventilation and surfactant. Of the 132 patients, 14 were excluded for a variety of reasons, including being left hospital before the final outcome was assessed (9), did not consent (10), had multiple congenital defects (3), and died after extensive resuscitation in the delivery room (1). As a result, 109 neonates were included and randomized in the present study: 54 in the intervention group (budesonide + surfactant), and 55 in the control group (surfactant). The study flow chart as per CONSORT guidelines has been shown in Figure 1. [14] The mean gestational age of the study group was $26.1 \pm 0.2$ weeks, and the mean birth weight was $770.5 \pm 31.5$ gms. The baseline characteristics were comparable between the groups (Table 1).

Table 1

Comparison of baseline characteristics among study groups.

\begin{tabular}{|llll|}
\hline Variables & $\begin{array}{l}\text { Intervention } \\
(\mathbf{n = 5 4 )}\end{array}$ & $\begin{array}{l}\text { Control } \\
(\mathbf{n = 5 5 )}\end{array}$ & P - value \\
\hline Birth wt. (Mean) & $778.1 \pm 46.7$ & $763.2 \pm 43.5$ & 0.644 \\
\hline Gestation Age & $26.3 \pm 0.4$ & $25.9 \pm 0.4$ & 0.245 \\
\hline Male & $29 / 54(53.7 \%)$ & $25 / 55(45.4 \%)$ & 0.445 \\
\hline SGA & $5 / 54(9.2 \%)$ & $4 / 55(7.2 \%)$ & 0.741 \\
\hline Antenatal betamethasone (2 doses) & $39 / 54(72.2 \%)$ & $40 / 55(72.7 \%)$ & 1.00 \\
\hline Chorioamnionitis & $3 / 54(5.5 \%)$ & $5 / 55(9 \%)$ & 0.716 \\
\hline Cesarean section & $38 / 54(70.3 \%)$ & $35 / 55(63.6 \%)$ & 0.542 \\
\hline DR - CPAP & $10 / 54(18.5 \%)$ & $11 / 55(20 \%)$ & 1.00 \\
\hline DR - Intubation & $44 / 54(81.4 \%)$ & $44 / 55(80 \%)$ & 1.00 \\
\hline APGAR at 1 min, median(range) & $5(1-8)$ & $5(1-9)$ & \\
\hline APGAR at 5 min, median(range) & $7(3-9)$ & $7(2-9)$ & \\
\hline Modified CRIB II, median (range) & $9(7-14)$ & $10(6-13)$ & \\
\hline SGA - Small for gestation age, DR - Delivery room, CPAP - Continuous positive airway pressure, & & \\
\hline CRIB - Clinical risk index for babies & & & \\
\hline
\end{tabular}


Primary Outcomes: Primary outcomes examined in this study were incidence of death, BPD, and combined BPD or death. Death (intervention group: 9/54 [16.6\%]; control group: 14/55 [25.4\%]; $p=0.267$ ) and combined BPD or death (intervention group: 41/54 [75.9\%]; control group: 47/55 [85.4\%]; $p=0.211$ ) both showed decreasing trends, which was not statistically significant. The incidence of BPD was similar in both groups (intervention group: 32/54 [59.2\%]; control group: 33/55 [60\%]; $p=0.937$ ) (Table 2).

Table 2

Primary outcomes in study groups.

\begin{tabular}{|c|c|c|c|c|c|}
\hline \multicolumn{2}{|l|}{ Variables } & Intervention $(n=54)$ & Control $(n=55)$ & $\mathrm{RR}(95 \% \mathrm{ci})$ & $P$ - value \\
\hline \multicolumn{2}{|l|}{ Death } & 9/54 (16.6\%) & $14 / 55$ (25.4\%) & $0.65(0.30-1.38)$ & 0.267 \\
\hline \multicolumn{2}{|l|}{ BPD } & $32 / 54(59.2 \%)$ & $33 / 55(60 \%)$ & $0.98(0.72-1.34)$ & 0.937 \\
\hline \multirow[t]{3}{*}{ BPD (severity) } & Mild & $20 / 54(37 \%)$ & $20 / 55(36.3 \%)$ & $1.01(0.62-1.66)$ & 0.941 \\
\hline & Moderate & $7 / 54(12.9 \%)$ & $2 / 55(3.6 \%)$ & $3.56(0.77-16.3)$ & 0.102 \\
\hline & Severe & $5 / 54(9.2 \%)$ & $11 / 55(20 \%)$ & $0.46(0.17-1.24)$ & 0.126 \\
\hline \multicolumn{2}{|c|}{ BPD (all) or Death } & $41 / 54(75.9 \%)$ & $47 / 55(85.4 \%)$ & $0.88(0.73-1.06)$ & 0.211 \\
\hline \multicolumn{2}{|c|}{ BPD (Severe) or Death } & $14 / 54(25.9 \%)$ & $25 / 55(45.4 \%)$ & $0.57(0.33-0.97)$ & 0.040 \\
\hline
\end{tabular}

Secondary Outcomes: The adverse effects due to steroids like hyperglycemia, hypertension requiring treatment, clinical sepsis or bacteraemia and morbidities related to prematurity like HsPDA, IVH, Periventricular leukomalacia (PVL), NEC, severe ROP (>stage 2) were all evaluated as secondary outcomes in the current study (Table 3). The intervention group had no notable adverse effects, such as hyperglycemia or hypertension. Both groups required inotropes, had PDA that required treatment, and had an equal rate of intestinal perforation and culture-positive sepsis. The intervention group had a significantly lower incidence of any grade IVH (intervention group: 12/54 [22.2\%]; control group: 24/55 [43.6\%]; $p=0.024$ ) as well as high-grade IVH ( $\geq$ grade 3 ) or PVL (intervention group: $5 / 54$ [9. \%]; control group: $14 / 55$ [25.4\%];p=0.041).Compared to thecontrol,the intervention group required significantly fewer inpatient days. (Intervention $76.5 \pm 10.2$ days vs. control $84.1 \pm 12.9$ days). 
Table 3

Secondary outcomes in study groups.

\begin{tabular}{|llll|}
\hline Variables & Intervention (n=54) & $\begin{array}{l}\text { Control } \\
(\mathbf{n = 5 5 )}\end{array}$ & P - value \\
\hline Hyperglycemia requiring Insulin & $1 / 54(1.8 \%)$ & $1 / 55(1.8 \%)$ & 1.000 \\
\hline Hypertension requiring treatment & $1 / 54(1.8 \%)$ & $3 / 55(5.4 \%)$ & 0.618 \\
\hline lonotropic requirement & $18 / 54(33.3 \%)$ & $26 / 55(47.2 \%)$ & 0.172 \\
\hline PDA requiring treatment & $27 / 54(50 \%)$ & $34 / 55(61.8 \%)$ & 0.249 \\
\hline Any IVH & $12 / 54(22.2 \%)$ & $24 / 55(43.6 \%)$ & 0.024 \\
\hline IVH $\geq$ Grade 3 or PVL & $5 / 54(9.2 \%)$ & $14 / 55(25.4 \%)$ & 0.041 \\
\hline ROP > Stage 2 & $6 / 54(11.1 \%)$ & $5 / 55(9 \%)$ & 0.761 \\
\hline Intestinal perforation (NEC/SIP) & $4 / 54(7.4 \%)$ & $5 / 55(9 \%)$ & 1.000 \\
\hline Culture positive sepsis & $15 / 54(27.7 \%)$ & $22 / 55(40 \%)$ & 0.225 \\
\hline $\begin{array}{l}\text { Duration of hospitalization } \\
\text { (In survivors, Mean) }\end{array}$ & $76.5 \pm 10.2$ & $84.1 \pm 12.9$ & 0.001 \\
\hline $\begin{array}{l}\text { PDA - Patent ductus arteriosus, IVH - Intraventricular haemorrhage, PVL - Periventricular } \\
\text { leukomalacia, ROP - Retinopathy of prematurity, NEC - Necrotising enterocolitis, SIP - Spontaneous } \\
\text { intestinal perforation }\end{array}$ & & \\
\hline
\end{tabular}


Table 4

Other respiratory outcomes in study groups.

\begin{tabular}{|llll|}
\hline Variables & $\begin{array}{l}\text { Intervention } \\
(\mathbf{n = 5 4 )}\end{array}$ & $\begin{array}{l}\text { Control } \\
(\mathbf{n = 5 5 )}\end{array}$ & $\mathbf{P}$ - value \\
\hline Surfactant Redosing & $10 / 54(18.5 \%)$ & $18 / 55(32.7 \%)$ & 0.124 \\
\hline Pulmonary Hemorrhage & $1 / 54(1.8 \%)$ & $4 / 55(7.2 \%)$ & 0.363 \\
\hline Pneumothorax & $1 / 54(1.8 \%)$ & $3 / 55(5.4 \%)$ & 0.618 \\
\hline Fio2 requirement at 24-HOL (In survivors) & $22.6 \pm 1.7$ & $29.9 \pm 3.5$ & 0.001 \\
\hline Fio2 weaned to 21\% at (DOL, In survivors) & $3.1 \pm 2.3$ & $14.9 \pm 7.4$ & 0.001 \\
\hline Duration of invasive ventilation & $5.3 \pm 2$ & $8.8 \pm 3.4$ & 0.001 \\
\hline Duration of non-invasive ventilation & $31.1 \pm 8.1$ & $35.2 \pm 8.9$ & 0.040 \\
\hline DOL at which no respiratory support / O2 & $51.6 \pm 15.8$ & $62.2 \pm 14.7$ & 0.003 \\
\hline Duration of caffeine given (Days) & $59.91 \pm 9.8$ & $70.5 \pm 12.6$ & 0.001 \\
\hline Use of Systemic postnatal steroids & $2 / 54(3.7 \%)$ & $5 / 55(9 \%)$ & 0.437 \\
\hline
\end{tabular}

\section{Other respiratory outcomes}

Respiratory outcomes between the intervention and control groups including need for surfactant redosing, Fio2 requirement, duration of invasive and non-invasive ventilation, duration of caffeine, use of postnatal systemic steroids for BPD and complications like pulmonary hemorrhage, pneumothorax were compared. The intervention group compared to control group had significantly lower $\mathrm{FiO}_{2}$ requirement at 24 hours of life $(22.6 \pm 1.7$ vs $29.9 \pm 3.5$, p-value $=0.001)$, and was weaned to $21 \% \mathrm{FiO}_{2}$ early $(3.1 \pm 2.3 \mathrm{~d}$ vs $14.9 \pm 7.4 \mathrm{~d}, \mathrm{p}=0.001)$. The total duration of respiratory support both invasive $(5.3 \pm 2$ vs. $8.8 \pm 3.4 \mathrm{~d})$ and non-invasive ventilation ( $31.1 \pm 8.1$ vs. $35.2 \pm 8.9 \mathrm{~d}$ ) was significantly less in intervention group as compared to control group. Two infants (3.7\%) in the intervention group and five infants (9\%) in the control group received systemic dexamethasone therapy.

\section{Discussion}

This study compared intratracheal surfactant and budesonide to surfactant alone for prevention of BPD in extreme preterm infants admitted to a tertiary level neonatal ICU. This is the first prospective, randomized, controlled superiority pilot trial conducted in an Indian population. Although there was a trend towards fewer deaths, combined death and BPD in the intervention group, there was no statistically significant difference between the two groups.

The use of intratracheal budesonide with surfactant provides direct anti-inflammatory actions at the target location, avoiding the inflammatory cascade and BPD. The use of intratracheal steroids to prevent 
BPD has been studied by Yeh et al., Pan et al., and Minji Heo et al. [8,15-17]. Previous studies by Yeh et al and pan et al used bovine surfactants $[8,15,16]$ and calf surfactant was used by Minji et al. [17] This study used a porcine surfactant (Curosurf) because it is feasible, requires less volume than other surfactants, is mixable (after mixing with budesonide, the mixture looks like a surfactant alone with no crystallization or discoloration), and has not been studied previously. Previous studies [8,15-17] studied VLBW babies. In this study we included only ELBW infants, the most vulnerable group with high incidence of BPD.

Unlike previous studies, which found that the intervention group had a lower incidence of BPD than the control group, in the current study, intervention group had a slightly greater incidence of BPD. One explanation is that the control group had more deaths, including neonates who may have had BPD if they had survived. The other two primary outcomes death and combined death or BPD were fewer in intervention group. However, these differences was not statistically significant. Heo et al. [17] reported similar results, however Yeh et al. [8,15] and Pan et al [16] found that the intervention group had a lower composite outcome of mortality or BPD than the control group.

One important observation in this study is that the combined outcome of death or severe BPD is significantly lower in the intervention group compared to the control group (RR 0.57(0.33-0.97); $p=0.040)$, indicating that, severe morbidity and mortality related to BPD have been shown to decrease significantly with the intervention used. Yeh et al. [8] observed similar findings (RR 0.61[0.41-0.91]; $p=0.016$ ), however another study [17] found non-significant decreasing trend (RR 0.18(0.02-1.39); $p=0.102)$. If comparable findings can be replicated in larger, multicentric trials, this easily accessible and low-cost intervention might be put into common clinical practice. This technique has the potential to change lung damage and result in considerable respiratory and overall health benefits.

The current study's total incidence of BPD, combined death or BPD were higher as compared to previous studies because our study population was more premature and smaller ( $<28$ weeks and ELBW) than previously studied ( $<32$ weeks and VLBW ) neonates. [8,15-17] Despite the high prevalence of BPD in the current study population, only two newborns in the control group were discharged with home oxygen, and the prevalence of ROP ${ }^{3}$ stage 2 was minimal (10\%).

The mechanism of action of budesonide is probably anti-inflammatory. Intratracheal surfactant and budesonide instillation showed immediate improvement in pulmonary status compared to systemic dexamethasone injections. [18] The direct local anti-inflammatory effect and the larger volume of instillation $(3.5 \mathrm{ml} / \mathrm{kg})$ in the intervention group compared to the control group may have enhanced surfactant and budesonide distribution. A prolonged effect on the lungs is implied by the fact that the intervention group required less FiO2 at 24 hours of birth, weaned to 21\% FiO2 earlier, and spent less time on respiratory support (invasive and non-invasive).Our findings suggested that budesonide was effective early in therapy, which may have translated to less oxygen and respiratory support, resulting in an earlier hospital discharge in intervention group (76.5 \pm 10.2 days vs $84.1 \pm 12.9$ days). 
Corticosteroids are known to induce hyperglycemia, hypertension, and an increased risk of infection, as well as long-term effects on physical development, neuromotor, and cognitive function. [19] In this study, we only examined the short-term side effects of steroids and observed no differences in serum glucose, blood pressure, or culture-positive sepsis among both groups.

The intervention group had less inotrope requirement than the control group in the current study (18/54 [33.3\%] vs. 26/55 [47.2\%], $p=0.172$ ). Similar to dexamethasone, budesonide can increase cardiac stroke volume while stabilizing blood pressure.[20] In the study of Yeh et al, the blood pressure of the intervention group was significantly higher than that of the control group on days 3-5.[15] The later effect of budesonide on blood pressure stability may benefit newborns.

The intervention group had a lower incidence of IVH (any grade) and high-grade IVH (grade 3 \&4) than the control group (12/54 [22.2\%] versus 24/55 [43.6\%], $p=0.24 ; 5 / 54$ [9.2\%] vs. 14/55 [25.4\%], $p=0.041)$. A lower IVH may be due to fewer ventilation days, less ionotropic requirement, and less hsPDA, all contributing to a stable course during the stay. However, the present study was not powered to look for these outcomes, and other variables were not examined. A larger study population is needed to evaluate these outcomes.

\section{Conclusion}

Intratracheal instillation of budesonide along with surfactant is feasible and safe. There was no difference in the incidence of death, BPD, combined BPD, or death among extreme preterm infants with RDS among the parallel groups. However, the combined outcome of death or severe BPD is significantly lower in extreme preterm infants who received intratracheal budesonide along with surfactant .

\section{Abbreviations}

BPD - Bronchopulmonary dysplasia

CPAP - continuous positive airway pressure

ELBW- Extreme low birth weight

IVH - Intraventricular haemorrhage

NEC - Necrotizing enterocolitis

NICU - Neonatal intensive care
PDA - Patent ductus arteriosus

PVL - Periventricular leukomalacia

RDS - Respiratory distress syndrome

ROP - Retinopathy of prematurity

VLBW - Very low birth weight

\section{Declarations}

Funding: This study did not receive any intramural or extramural funding. 
Conflict of interest: The authors declare that they have no conflicts of interest to declare associated with this work.

Availability of data and materials: If any further data regarding our work are needed we shall provide that. Code availability: Not applicable

Authors Contributions: KV , NP and DC involved in planning ,analysis and manuscript writing and editing of this study. VJ and AV involved in patient management and manuscript writing. All authors approved manuscript of this study.

Ethics Approval: The protocol of this study was reviewed and approved by Rainbow Hospital institutional ethics committee (RCHBH/081/03-2018).

Consent to participate: Written informed consent was obtained from the parents

Consent for publication: Not applicable

Acknowledgments: We are thankful to parents of neonates for their co-operation and also acknowledge contribution of neonatal trainee doctors and nursing staff.

\section{References}

1. McEvoy CT, Aschner JL (2015 Dec) The Natural History of Bronchopulmonary Dysplasia: The Case for Primary Prevention. Clin Perinatol 42(4):911-931

2. Jensen EA (2019) Prevention of Bronchopulmonary Dysplasia: A Summary of Evidence-Based Strategies. NeoReviews. Apr 1;20(4):e189-201

3. Speer CP (2004) Pre- and postnatal inflammatory mechanisms in chronic lung disease of preterm infants. Paediatr Respir Rev 5(Suppl A):S241-244

4. Doyle LW, Ehrenkranz RA, Halliday HL (2014 May) Early ( 8 days) postnatal corticosteroids for preventing chronic lung disease in preterm infants. Cochrane Database Syst Rev 13(5):CD001146

5. Brattsand R, Thalén A, Roempke K, Källström L, Gruvstad E (1982) Development of new glucocorticosteroids with a very high ratio between topical and systemic activities. Eur $\mathrm{J}$ Respir Dis Suppl 122:62-73

6. Miller-Larsson A, Mattsson H, Hjertberg E, Dahlbäck M, Tunek A, Brattsand R (1998 Jul) Reversible fatty acid conjugation of budesonide. Novel mechanism for prolonged retention of topically applied steroid in airway tissue. Drug Metab Dispos Biol Fate Chem 26(7):623-630

7. Bassler D, Plavka R, Shinwell ES, Hallman M, Jarreau P-H, Carnielli V et al (2015 Oct) Early Inhaled Budesonide for the Prevention of Bronchopulmonary Dysplasia. N Engl J Med 15(16):1497-1506

8. Yeh TF, Chen CM, Wu SY, Husan Z, Li TC, Hsieh WS et al (2016) Intratracheal Administration of Budesonide/Surfactant to Prevent Bronchopulmonary Dysplasia. Am J Respir Crit Care Med. Jan 
1;193(1):86-95

9. Chen C-M, Huang L, Wang M, Yeh T (2014 Oct) PS-286 Surfactant (s) Supplemented With Budesonide (b) For Prevention Of Bronchopulmonary Dysplasia - Biophysical And Chemical Stability Of S/b Mixture. Arch Dis Child 199:A215-A215

10. Doyle LW, Davis PG, Morley CJ, McPhee A, Carlin JB, DART Study Investigators (2006 Jan) Low-dose dexamethasone facilitates extubation among chronically ventilator-dependent infants: a multicenter, international, randomized, controlled trial. Pediatrics 117(1):75-83

11. Jobe AH, Bancalari E (2001 Jun) Bronchopulmonary dysplasia. Am J Respir Crit Care Med 163(7):1723-1729

12. Sealed Envelope Ltd (2012) Power calculator for binary outcome superiority trial. Available from: https://www.sealedenvelope.com/power/binary-superiority/

13. Corp IBM Released 2020. IBM SPSS Statistics, Version 27.0. Armonk, NY:IBM Corp

14. Eldridge SM, Chan CL, Campbell MJ, Bond CM, Hopewell S, Thabane L et al (2016) CONSORT 2010 statement: extension to randomised pilot and feasibility trials. Pilot Feasibility Study 2:64

15. Yeh TF, Lin HC, Chang CH, Wu TS, Su BH, Li TC et al (2008 May) Early intratracheal instillation of budesonide using surfactant as a vehicle to prevent chronic lung disease in preterm infants: a pilot study. Pediatrics 121(5):e1310-1318

16. Pan J, Chen M-W, Ni W-Q, Fang T, Zhang H, Chen Y et al (2017 Feb) [Clinical efficacy of pulmonary surfactant combined with budesonide for preventing bronchopulmonary dysplasia in very low birth weight infants]. Zhongguo Dang Dai Er Ke Za Zhi Chin J Contemp Pediatr 19(2):137-141

17. Heo M, Jeon GW (2020) Intratracheal administration of budesonide with surfactant in very low birth weight infants to prevent bronchopulmonary dysplasia. Turk J Pediatr 62(4):551-559

18. Yeh TF, Torre JA, Rastogi A, Anyebuno MA, Pildes RS (1990 Aug) Early postnatal dexamethasone therapy in premature infants with severe respiratory distress syndrome: a double-blind, controlled study. J Pediatr 117(2 Pt 1):273-282

19. Halliday HL (2017) Update on Postnatal Steroids. Neonatology 111(4):415-422

20. Wu JM, Yeh TF, Wang JY, Wang JN, Lin YJ, Hsieh WS et al (1999) The role of pulmonary inflammation in the development of pulmonary hypertension in newborn with meconium aspiration syndrome (MAS). Pediatr Pulmonol Suppl 18:205-208

\section{Figures}



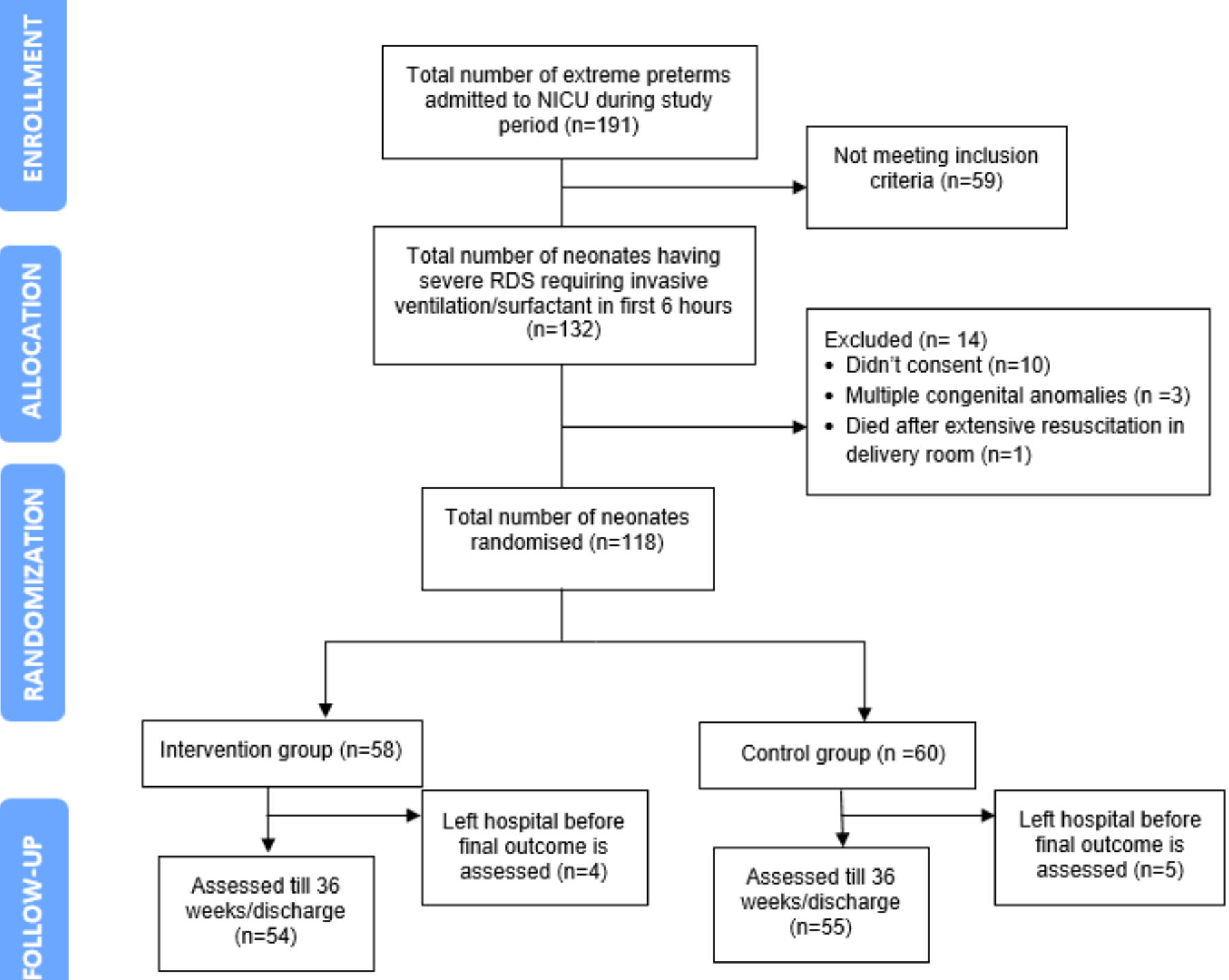

Figure 1

Study flow chart. [14]

RDS = respiratory distress syndrome 